\title{
Branched endografts for thoracoabdominal aneurysms
}

\author{
Roy Greenberg, MD, Matthew Eagleton, MD, and Tara Mastracci, MD
}

Purpose: Endovascular management of thoracoabdominal aneurysms has been studied since 2001, with marked advances allowing for the treatment of complex anatomic situations including chronic aortic dissections, tortuous anatomy, and extensive aneurysms that involve the visceral segment, aortic arch, and iliacs as well. However, the technology is not widely disseminated, and a thorough understanding of the engineering principles, imaging techniques, and devices available is required.

Methods: Reinforced fenestrated branches coupled with balloon expandable stent grafts, and side-arm branch designs mated with self-expanding stent grafts have been used. Pure fenestrated designs were used for juxtarenal aneurysms, whereas thoracoabdominal aneurysms were treated with reinforced fenestrated branches or hybrid devices including side-arm branches and reinforced fenestrated branches. Intraoperative fusion techniques have been used since 2009, whereby preoperative computed tomographic data are fused with intraoperative fluoroscopy. Long-term survival in accordance with extent of disease was assessed with life table analysis techniques, and differences were analyzed using the log rank test. Intermediate-term data pertaining to patency related to both types of branches and paraplegia have been evaluated and previously published.

Results: A total of 406 patients with thoracoabdominal aneurysms and 227 patients with juxtarenal aneurysms have been enrolled in a prospective study. Perioperative and 2-year survival were most closely related to extent of initial disease and were estimated to be $1.8 \%$ and $82 \%$ for juxtarenal aneurysms, $2.3 \%$ and $82 \%$ for type IV, and $5.2 \%$ and $74 \%$ for type II and III thoracoabdominal aneurysms at 24 months, respectively. When patients undergoing endovascular repair (ER group) were matched with those having contemporary surgical repair (SR group) for anatomic disease extent, mortality was similar at 30 days $(5.7 \%$ ER vs $8.3 \%$ SR; $P=.2)$ and at 12 months $(15.6 \%$ ER vs $15.9 \%$ SR; $P=.9)$. Paraplegia risk was also similar between the 2 groups $(4.3 \%$ ER vs $7.5 \%$ SR, respectively; $P=.08)$. Among the 633 patients, there were $5(0.8 \%)$ late ruptures at a mean of 18 months after treatment, of which 4 were fatal. They were attributed to component separation $(\mathrm{n}=3)$, a remote aneurysm rupture proximal to the endovascular repair, and a failed surgical polyester graft distal to the repair. Reinforced fenestrated branch patency, when coupled with balloon-expandable stent grafts, was $97.8 \%$ at a mean follow-up of 15 months. Side-arm branch occlusion occurred in only 1 case, within 24 hours of the procedure. New imaging tools resulted in a marked reduction in the average contrast dose $(>50 \%)$.

Conclusions: Intermediate-term results with multiple methods of endovascular repair of thoracoabdominal aneurysm indicate the technical feasibility of the procedure and show great promise in patients considered at high risk for open surgery. The intermediate-term patency and survival are excellent, and ruptures are exceedingly uncommon. However, mortality and spinal cord ischemia risks are still considerable with this technique. (J Thorac Cardiovasc Surg 2010;140:S171-8)

The management of patients with complex diseases is a balance of clinical risks. The benefit of any type of intervention, surgical or endovascular, must be weighed against the risk of aneurysm rupture if left untreated. Additionally, one must

\footnotetext{
From the Departments of Vascular Surgery, Cardiothoracic Surgery, Radiology, and Biomedical Engineering, Cleveland Clinic Foundation, Cleveland, Ohio.

Disclosures: Roy Greenberg receives grant/research support from Cook, Inc, Gore \& Associates, Inc, and Terarecon; is a consultant for Cook, Inc; and holds intellectual property rights with Cook, Inc. Tara Mastracci received consulting fees from Cook Medical. Matthew Eagleton receives consulting fees from Bolton Medical.

Received for publication May 12, 2010; accepted for publication July 20, 2010.

Address for reprints: Roy Greenberg, MD, Director, Endovascular Research, Cleve-

land Clinic Foundation, Desk F-30, 9500 Euclid Ave, Cleveland, Ohio 44195

(E-mail: greenbr@ccf.org).

$0022-5223 / \$ 36.00$

Copyright (c) 2010 by The American Association for Thoracic Surgery

doi:10.1016/j.jtcvs.2010.07.061
}

consider the effect a given intervention may have on the quality of a patient's life. Despite numerous single-center series defining the risks of mortality, spinal cord ischemia, and renal events when procedures are done at centers of excellence, ${ }^{1-4}$ little scientific data exist to help clinicians with these difficult decisions. In fact, statewide audits have reported alarmingly high mortality rates at 1 month $(19 \%)$ and 1 year $(31 \%)$ when such operations are undertaken at any hospital. ${ }^{5}$ Proper decision-making requires an understanding of the interventional options (open versus endovascular surgery), perioperative management, follow-up strategies, and the effect of any patient-related risk factors on the development of complications. The implementation of an endovascular approach requires further knowledge regarding device engineering issues, a solid foundation in imaging techniques, and surgical skills. 


\section{Abbreviations and Acronyms \\ CT = computed tomography \\ $\mathrm{ER}$ group $=$ endovascular repair group \\ $\mathrm{SR}$ group $=$ surgical repair group}

\section{BRANCHED DEVICE TECHNOLOGIES Reinforced Fenestrations}

The first and conceptually simplest branches are termed reinforced fenestrations (Figure 1). These are essentially holes, customized to the size and location of the target visceral vessels, which are placed in the aortic graft and reinforced with a circumferential nitinol wire. After insertion and device orientation, the delivery sheath is withdrawn, allowing the aortic stent graft to partially expand within the aorta. The distal aspect of the graft is then accessed via the contralateral groin, and, from within the aortic device, the fenestration and target vessels are sequentially catheterized. During this period, the aortic stent graft position may be adjusted longitudinally or radially, given control offered by the proximal and distal attachment of the device to the delivery system and the fact that the aortic graft fabric is only partially expanded. Sheaths are introduced into each branch, and the wire that is partially constraining the aortic stent graft diameter is removed, allowing full expansion of the device. The target visceral arteries are then mated to the aortic device with balloon-expandable stent grafts. Fenestrations can be placed in the top portion of an abdominal device, the distal portion of a thoracic device, or the proximal portion of the thoracic device to be used to accommodate the arch vessels. Additional components, such as thoracic grafts and distal bifurcated or iliac branched devices, are then placed to complete the repair.

\section{Side-Arm Branches}

Two general options exist for side-arm branches. Side branches can be used for all 4 visceral vessels, or a hybrid device can be created with side-arm branches and fenestrations (Figure 2). The 2 fundamental types of side-arm branches relate to their length and angulation in relation to the aorta. Both types of branches are mated with selfexpanding stent grafts (Fluency; C. R. Bard, Inc, Murray Hill, NJ; or Viabahn; W. L. Gore \& Associates, Inc, Flagstaff, Ariz). Side-arm branches are accessed from above, often over a preloaded wire that is snared from an axillary artery access site. Sheaths are then advanced into the sidearm branch, and a separate puncture in the sheaths allows for a second wire and catheter to be placed to gain access into the target branch. The mating stent grafts are then placed in the desired location. Supplemental support may be necessary and is achieved with additional stents when required (Wallstent; Boston Scientific, Natick, Mass; or Genesis;
Cordis Endovascular, Great Lakes, NJ). Standardized devices that fit most type I, II, or III thoracoabdominal aneurysms are now available for patients that meet certain anatomic criteria for each type of device.

\section{Device Design and Anatomic Patient Selection}

High-resolution computed tomographic (CT) scans of the entire chest, abdomen, and pelvis are required to ensure that patients meet the standardized device criteria or to make measurements for customized devices. Centerline of flow technology is used to determine accurate longitudinal relationships between the visceral or supra-aortic trunk vessels. The radial orientation of the branch is determined from images generated orthogonal to the aortic centerline of flow. These techniques are relatively simple but require the use of a 3-dimensional visualization program or workstation. Reinforced fenestrations were preferentially used for all juxtarenal and type IV thoracoabdominal aneurysms. Helical side-arm branches were used for one or both of the visceral vessels in type II and III thoracoabdominal aneurysms when the lumen size was greater than $35 \mathrm{~mm}$ and there was no dissection. Reinforced fenestrations were used for all renal arteries, even when side-arm branches were used for the visceral vessels, as the renal arteries in extensive thoracoabdominal aneurysms tend to angle cranially. ${ }^{6}$

\section{Operating Rooms and Imaging Systems}

The operative team included the surgeon-interventionalist, a resident or fellow, a scrub nurse, a circulating nurse, and a radiology technician, all facile in wire and catheter technology. The anesthetic team for such cases must be competent in placement of cerebrospinal fluid drainage catheters in addition to transesophageal echocardiography. Fixed imaging systems with integrated cooling systems and floating point tables were used for all cases. Recent advances in imaging technologies have made the branched endograft procedure much simpler, with the added benefit of lower contrast doses. Much of this relates to the ability to integrate a preoperative CT scan into the operative fluoroscopic image, as well as to obtain intraoperative CT images (Figure 3). These techniques facilitate the implantation procedure by allowing for the early detection of type I or III endoleaks, for more accurate positioning of the aortic device in relation to critical branches without the need for angiography, and by simplifying the skills required to access the target vessels using a defined road map. The latter 2 techniques are accomplished with the aid of a CT scan, performed after preparing and draping the patient, which serves as a means to register other studies in 3 dimensions. The preoperative high-resolution contrast-enhanced preoperative CT scan is registered with the intraoperative noncontrast CT scan using boney landmarks. Thus, the preoperative study is imported into the operating room and registered with the patient position, table position, and in relation to the imaging intensifier and 


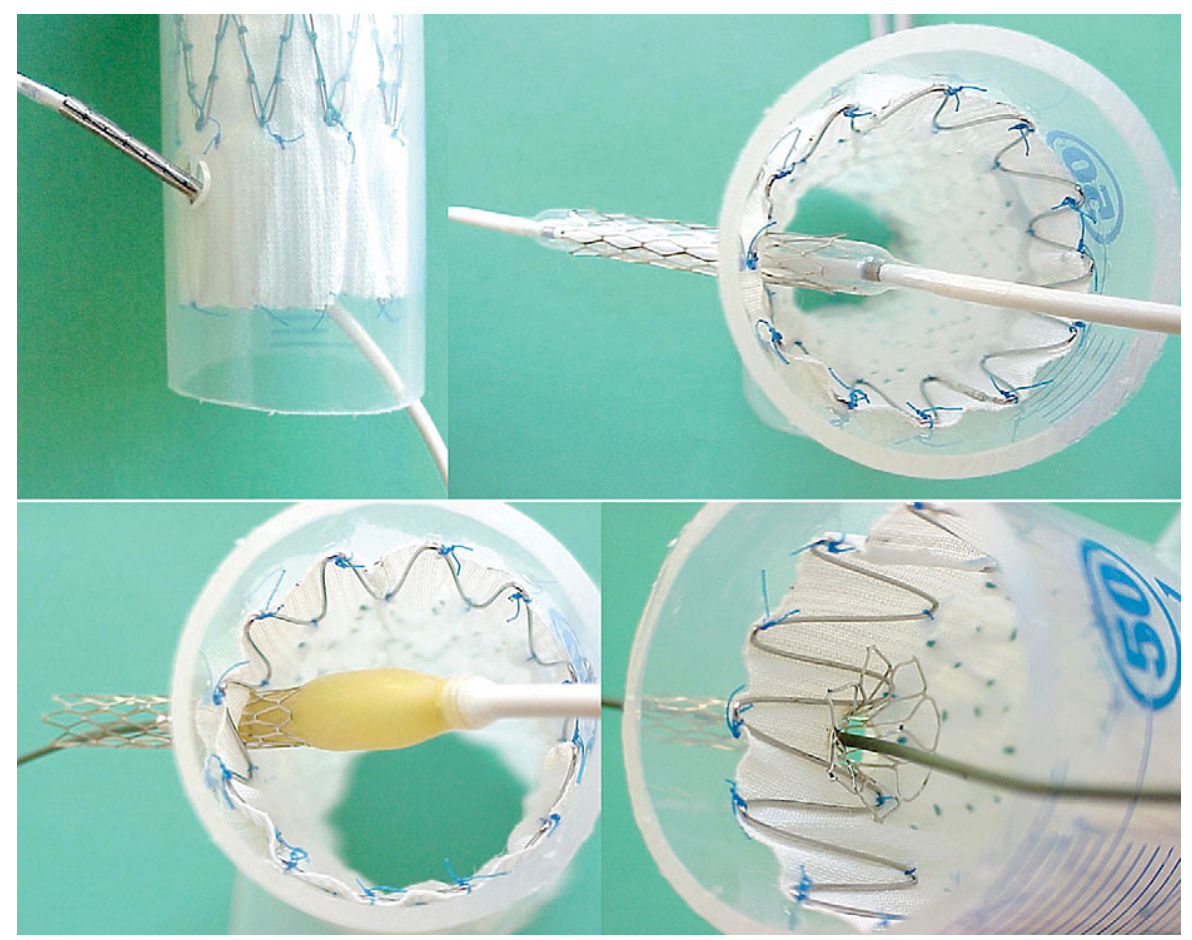

FIGURE 1. A fenestration placed in the distal end of a thoracic device is illustrated here. There is a balloon-expandable stent placed through the fenestration in the first panel, and inflated to a diameter matching the target vessel in the second panel. A larger balloon is then placed into the aortic portion of the stent and used to flare the stent against the aortic graft wall. Such a procedure can be done with a balloon-expandable stent graft in the same manner as illustrated here.

generator. Marking tools based on an imaging workstation can then be used to highlight each of the target vessels, which are then easily visualized without the addition of any contrast.

\section{RESULTS}

A physician-sponsored investigational devices exemption study was initiated in 2001. Early devices to treat juxtarenal aneurysms were limited to fenestrated designs mated with uncovered balloon-expandable stents. In 2004, reinforced fenestrations were used in conjunction with balloonexpandable stent grafts, and in 2005, side-arm branches for the visceral segment were added. A total of 633 patients were enrolled through March of 2010, 406 of whom were treated for thoracoabdominal aneurysms. The early experience was dominated by suprarenal or type IV thoracoabdominal aneurysms, and as the devices and procedural techniques evolved, a greater percentage of type II and III thoracoabdominal aneurysms were treated. Initial relative contraindications to endovascular treatment, such as tortuosity and chronic aortic dissection, were, over time, overcome by specific modifications to the delivery systems and device designs. Survival data in accordance with disease extent are depicted in Table 1 and Figure 4. Late ruptures were uncommon, with an overall incidence of $0.8 \%$. All rupture events are detailed in Table 2. Other specifics relating to the method by which repairs were classified, specific complications, and other technical issues have been previously published. ${ }^{1,7}$

\section{Branch Patency}

Two types of branches were used during thoracoabdominal aneurysms repair: helical side-arm branches for the celiac and superior mesenteric artery in type II and III aneurysms and reinforced fenestrations for all renal as well as the visceral vessels in patients with type IV aneurysms. Results regarding the patency and complications associated with renal fenestrations were published in $2009 .^{7}$ In summary, an incidence of occlusion of $2.2 \%$, with a mean follow-up of 15 months was noted in the 231 renal arteries joined to the aortic component with balloon-expandable stent grafts. The mean time to occlusion was about 3 months, and there were no late occlusions (beyond 12 months). Worsening glomerular filtration rate was noted in 4 of the 5 patients with renal occlusions, and 2 patients required hemodialysis, 1 permanently. With respect to helical side-arm branches, there has been only 1 occlusion: it occurred in the immediate perioperative period as a result of side-arm branch compression, and the patient died as a result. All other helical visceral branches have remained patent throughout follow-up.

\section{Mortality and Spinal Cord Ischemia}

A comparative study of patients undergoing open surgical repair (SR group) versus endovascular repair (ER group) treated for thoracic and thoracoabdominal aneurysms was published in $2008 .{ }^{1}$ When considering only patients with 

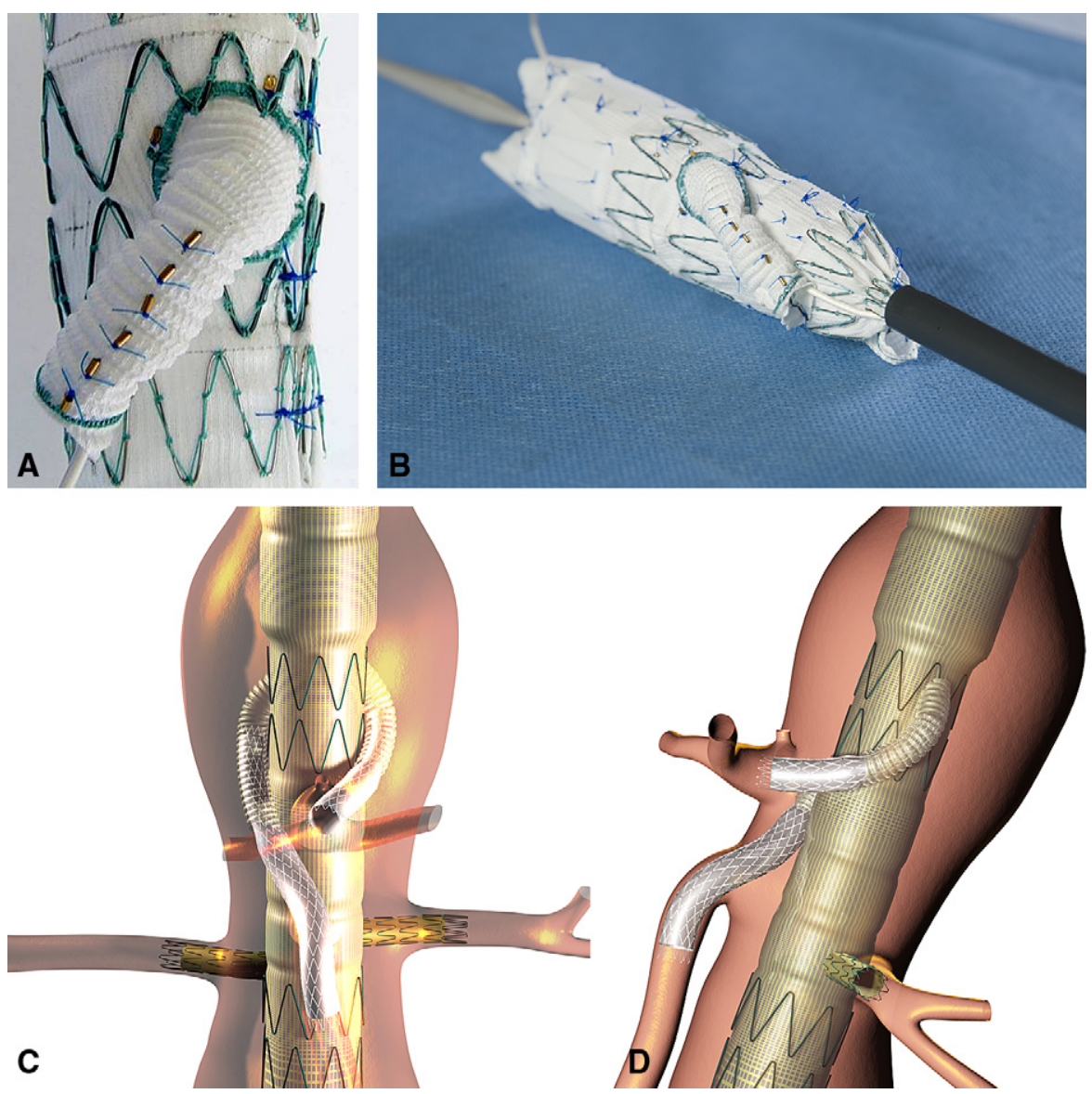

FIGURE 2. A, The graft image depicts a helical side-arm graft. The gold markers along the length of the side-arm coupled with the reinforcement rings proximally and distally facilitate orientation and access. The length of the side-arm is approximately $2.7 \mathrm{~cm}$, providing considerable overlap. The branch is preloaded with a catheter and wire that transcend the delivery system and are snared from the axillary access site to provide simple access from the arm into the branch. The helical side-arms were used preferentially for the visceral vessels in this study to minimize the distance and angulation between the branch exit and target vessel origin. Renal arteries, which are frequently upward pointing in type II and III thoracoabdominal aneurysms, were treated with reinforced fenestrations mated with balloon expandable stent grafts (images C and D for anterior and lateral views, respectively).

thoracoabdominal aneurysms, the ER patients were an average of 9 years older and sicker than the open SR cohort by every metric. The extent of the required repair and severity of any neurologic deficit were classified for both groups using a standardized method. Mortality was evaluated through 1 year of follow-up.

Similar rates of spinal cord ischemia were noted between the groups $(4.3 \%$ vs $7.5 \%$ for ER and SR respectively; $P=.08)$, and similar risks of mortality were also noted at 30 days $(5.7 \%$ vs $8.3 \%$ for ER and SR, respectively; $P=.2)$ and 12 months $(15.6 \%$ vs $15.9 \% ; P=.9)$. Anatomic extent of disease was by far the strongest predictor of the risk of developing a neurologic deficit, and type I and II thoracoabdominal aneurysms fared the worst in both groups. Interestingly, compromised iliac circulation or prior abdominal aortic aneurysm repair was associated with an increased risk of spinal cord ischemia for only the ER group, implying that such issues are likely offset by the ability to reimplant intercostal arteries. Although differences in comorbidities and the extent of treated disease existed between the 2 groups, the authors concluded that both means of repair are feasible and should be selectively applied to patients with thoracoabdominal aneurysms that require repair.

\section{DISCUSSION}

Clearly endovascular repair of thoracoabdominal aneurysms has certain advantages and disadvantages compared with conventional open surgical approaches. The advantages relate to the lack of invasiveness, which most profoundly mitigates the pulmonary risks, allowing for the treatment of patients who would otherwise be relegated to medical therapy alone. On the basis of this study, survival at 24 and 48 months is quite respectable, particularly in light of the high-risk population treated. These data, combined with the general benefits of shorter hospital stays, fewer days in the intensive care unit, and less of an adverse impact 

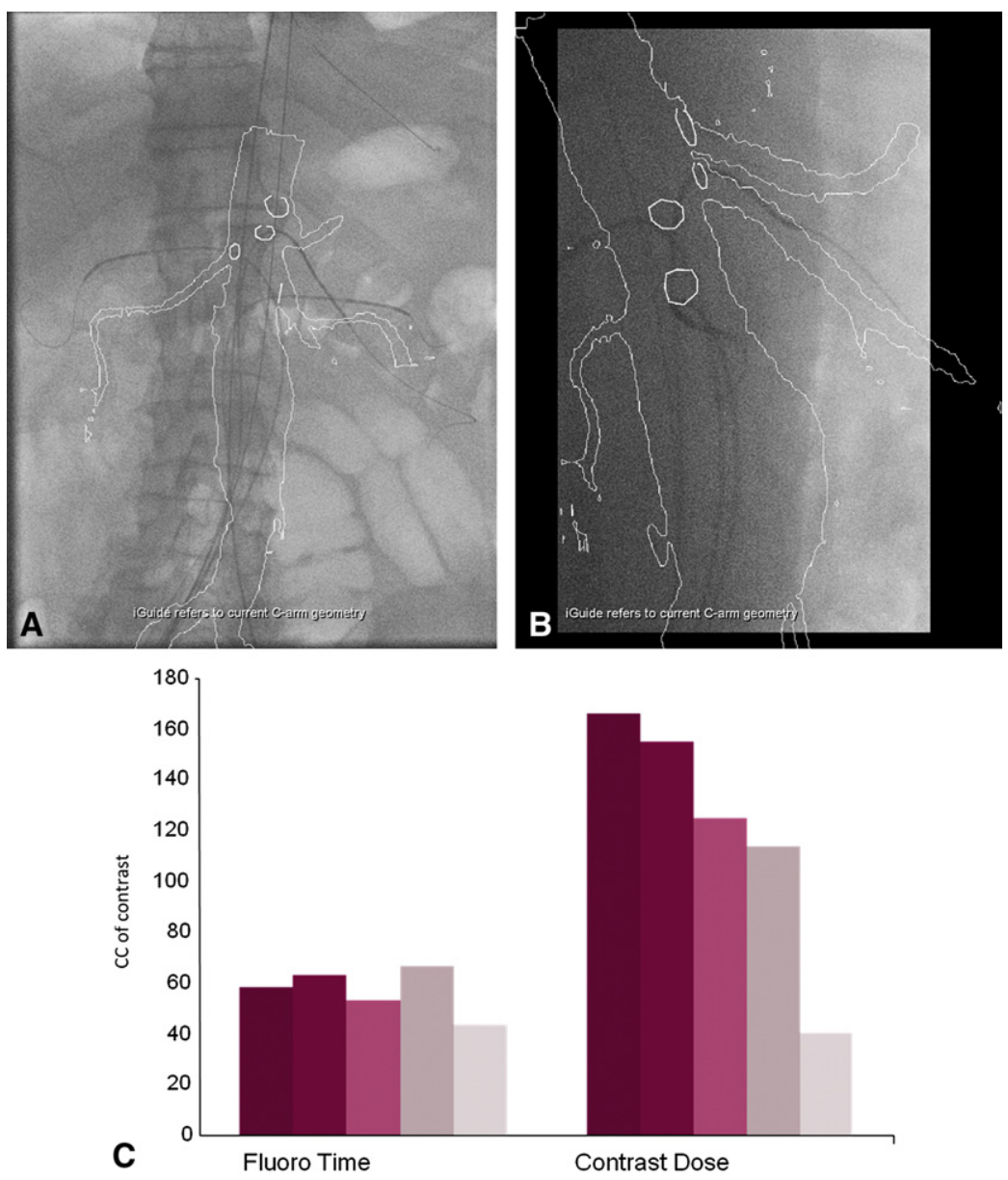

FIGURE 3. The preoperative CT scan was imported into the angio operative suite and fused with the fluoroscopic imaging system by registering it with a low-resolution noncontrast intraoperative CT scan. This was then used to trace out the visceral vasculature such that the vessels are easily cannulated using anterior (A) or lateral views (B). This resulted in a marked decrease in the amount of contrast required to perform these procedures. C depicts the average fluorscopic time and contrast dose for endovascular repair of type IV thoracoabdominal aneurysm on an annual basis, with the last column indicating contrast dose since we began using fusion imaging.

on the quality of life, likely parallel the benefits demonstrated in other series with less complex repairs. ${ }^{8-10}$

The placement of branched devices requires knowledge of 2 fundamental techniques, one used for the placement of balloon-expandable stent grafts within fenestrations, and the other relating to the placement of self-expanding stent grafts in side-arm branches, in addition to experience with aortic endografting and endovascular treatment of visceral vessel disease. Side-arm branches are usually coupled with preloaded wires and catheters, allowing sheaths to be directly advanced from above into the aortic device lumen or branch proper. Such delivery systems are not required for fenestrations, but a newer version of a fenestrated device will be available shortly that will include preloaded catheters and wires, eliminating the need to cannulate the aortic graft and fenestration from the contralateral groin.

In our series, the use of side-arm branches was limited to patients with extensive aneurysms and large lumen. The fundamental concept with the helical side-arm branches we use involves minimization of the distance between the branch

TABLE 1. Survival estimates, using life-table techniques in accordance with the extent of treated disease

\begin{tabular}{lcccc}
\hline Extent of Repair & No. of patients & Periop death (30 d) & Estimated survival 24 mo & Estimated survival 48 mo \\
\hline Total & 633 & $3.2 \%$ & & \\
Juxtarenal & 227 & $1.8 \%$ & $82 \%$ & $65 \%$ \\
Type I TAAA & 16 & $12.5 \%$ & $70 \%$ & N/A \\
Type II or III TAAA & 172 & $5.2 \%$ & $74 \%$ & $59 \%$ \\
Type IV TAAA & 218 & $2.3 \%$ & $82 \%$ & $70 \%$ \\
\hline
\end{tabular}

TAAA, Thoracoabdominal aneurysm; $N / A$, not available. 


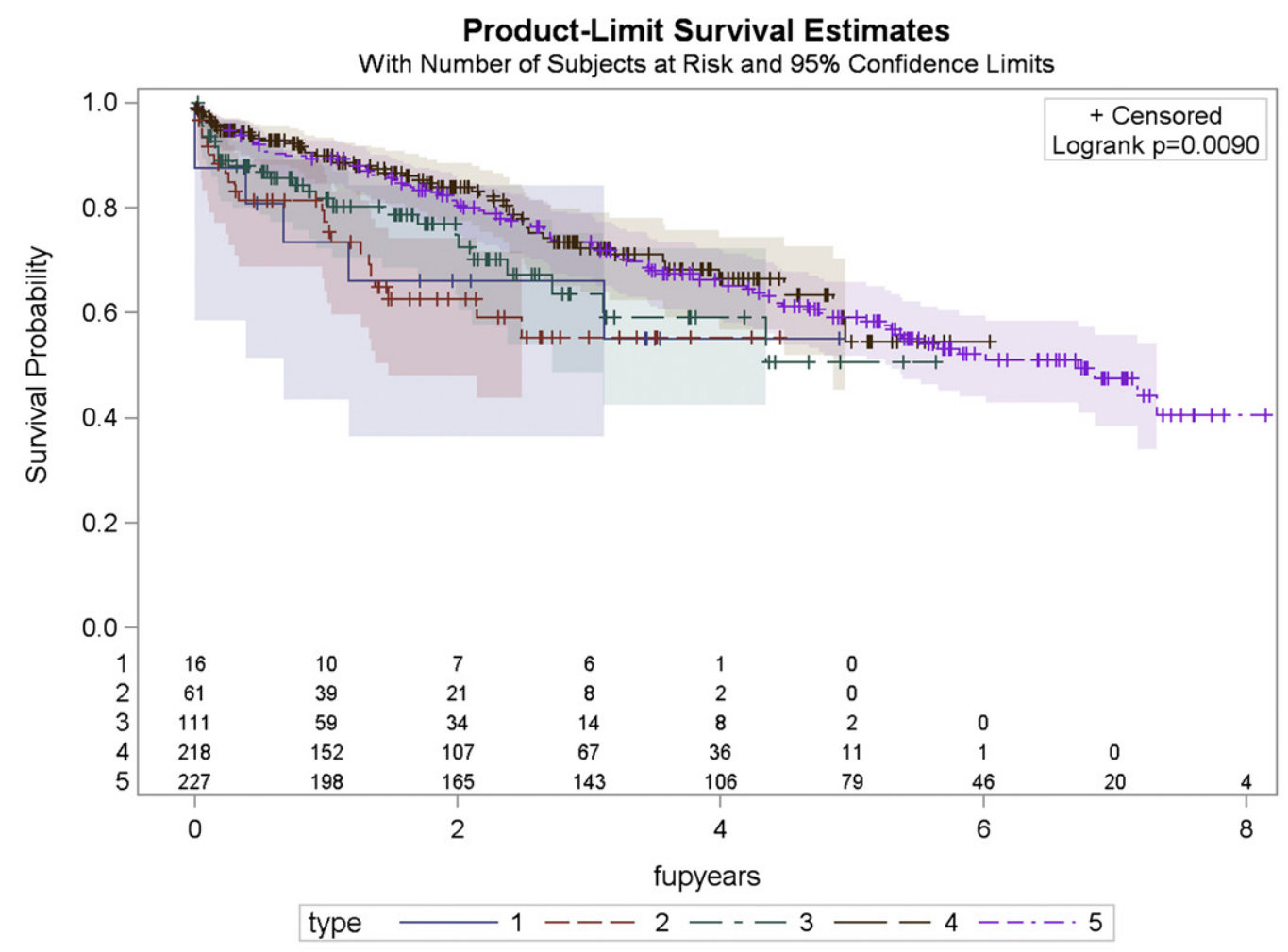

\begin{tabular}{|l|c|c|c|c|c|c|c|c|c|}
\hline CCF categories & Baseline & $1 \mathrm{~m}$ & $6 \mathrm{~m}$ & $1 \mathrm{y}$ & $2 \mathrm{y}$ & $3 \mathrm{y}$ & $4 \mathrm{y}$ & $5 \mathrm{y}$ & $6 \mathrm{y}$ \\
\hline Type 1 & 16 & 14 & 14 & 10 & 9 & 6 & 1 & 0 & 0 \\
\hline Type 2 & 61 & 58 & 50 & 42 & 25 & 13 & 3 & 0 & 0 \\
\hline Type 3 & 111 & 109 & 90 & 68 & 50 & 21 & 11 & 4 & 1 \\
\hline Type 4 & 218 & 212 & 189 & 164 & 129 & 85 & 51 & 20 & 4 \\
\hline Fen repair & 227 & 190 & 180 & 166 & 148 & 117 & 88 & 58 & 22 \\
\hline
\end{tabular}

FIGURE 4. Life table analyses with $95 \%$ confidence intervals were constructed for each type of aneurysm treated (type I, II, III, IV, and juxtarenal). In the figure, curve 5 indicates the survival curve for juxtarenal aneurysms. $C C F$, Cleveland Clinic Foundation.

origin and target vessel, coupled with the elimination of any angulation between the mating self-expanding stent graft and the target vessel (Figure 5). When met, such objectives will mitigate any strain placed on the mating stent graft device by ensuring that the path to the target vessel is straight and short. An additional benefit of this design is that it facilitates access during deployment. However, some disadvantages to this design exist as well. Helical branches are placed external to the tubular aortic component and thus require a reasonably large lumen to avoid side-branch compression. The single case of side-branch occlusion occurred as a result of compression along a segment of tortuous aorta and prompted the development of nitinol reinforcement wires along the length of the side-arm branch that will soon be implemented. Additionally, the packing density of side branches that are not oriented longitudinally is greater than side-arms that are straight up and down. This means that the delivery system may be larger for a helical branch. However, both types of branches seem to work quite well, and device iterations for both devices continue. ${ }^{1,11,12}$

The development of these techniques was initiated with the application of such devices to juxtarenal aneurysms (for fenestrated branches) and to preserve antegrade flow into internal iliac arteries in the setting of common iliac aneurysms (for side-arm technology). As we gained experience with these 2 types of procedures, we became progressively 
TABLE 2. Ruptured aneurysms after endovascular treatment of the 633 patients

\begin{tabular}{llcll}
\hline Patient No. & Aneurysm type & Time to rupture & Description & Outcome \\
\hline 1 & Juxtarenal & $28 \mathrm{mo}$ & Component separation, bifurcate from tubular treated surgically & Death \\
2 & Juxtarenal & $6 \mathrm{mo}$ & No information, data from death certificate only & Death \\
3 & Juxtarenal & $36 \mathrm{mo}$ & $\begin{array}{c}\text { Component separation, bifurcate from tubular treated } \\
\text { endovascularly }\end{array}$ & Survived \\
4 & Type III TAAA & $15 \mathrm{mo}$ & Old surgically placed Dacron graft rupture at interface with distal & Death \\
5 & Type III TAAA & $4 \mathrm{mo}$ & Arch aneurysm remote from endovascular repair & Death \\
\hline
\end{tabular}

TAAA, Thoracoabdominal aneurysm.

more aggressive with regard to the extent of the aneurysms we would treat. Such an approach with regard to technique dissemination makes sense. Interested parties should first gain proficiency with conventional abdominal aortic and thoracic aneurysm repairs and then use fenestrated devices for juxtarenal aneurysms and side-arm branches for iliac aneurysms. In both cases, surgical salvage is possible, and the loss of a single target vessel is not likely to be fatal. Additionally, advanced imaging techniques can be learned and teams can be established during this time period. Clinicians can then decide when it is appropriate to tackle more challenging cases that are likely to have no viable (survivable) bailout method should the endovascular approach be unsuccessful.

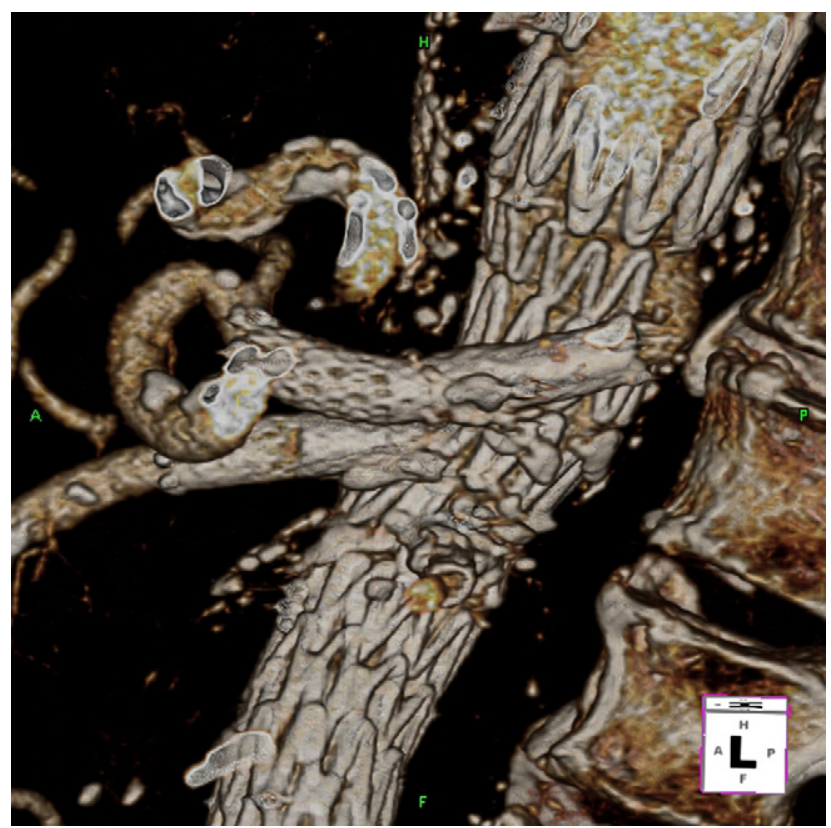

FIGURE 5. This lateral view of a 3 -dimensional reconstruction of a CT scan depicts a helical branch for the celiac artery. The mating stent graft (a Fluency graft) is straight, arising from the posterior aspect of the aortic graft, traversing in a slightly caudal angle, and landing with $2 \mathrm{~cm}$ of overlap into the origin of the celiac artery. This type of branch minimizes the angulation taken by the mating stent graft by allowing the helical portion attached to the aortic graft to assume the majority of the required angulation.
Much like other forms of endovascular treatment, the presence of intermediate-term results is reassuring but the lack of long-term data allows for skepticism. One must remember that the entire field of endovascular thoracoabdominal aneurysm repair is in flux, and therefore one must be cautious about any assumptions that the performance of one type of device can be applied to newer device iterations. Any marked change in the fabric or stent structure of a device will likely reset the follow-up clock to zero. This series includes over 600 patients treated with branched devices, with over 150 patients available for follow-up at 48 months. There are few, if any, surgical series that provide the extent or rigor of follow-up details for patients with thoracoabdominal aneurysms.

The future calls for prospective multicenter trials with the intent of evaluating the various devices and techniques. Despite the desire to obtain prospective randomized data, we remain pessimistic about the prospect of obtaining such information in the immediate future, given the patient population, current device constraints, and the rapid evolution of the devices and techniques. Lower profile devices, better delivery systems, and improved mating technologies are the subject of many research and development projects. Additionally, parallel advances in imaging technology have already facilitated complex endovascular procedures such as thoracoabdominal aneurysm repair as well as endovascular aortic valve placement. Fusion technology and mathematical modeling techniques allowing for image overlays, gating of preoperative high spatial and temporal resolution CT scans, along with the potential to deform the arterial images based on the mechanical characteristics of the arteries and endovascular tools, will reduce procedure length, complexity, contrast, and radiation dose. One can even postulate that the application of global positioning systems to such devices, when combined with the aforementioned advances, will bring us to another level of competence.

The series presented in this paper of 406 thoracoabdominal aneurysms overlaps with prior publications and does not include all of the details required to understand the risks and benefits of this technique, but it clearly illustrates its feasibility and broad applicability. The data also highlight that the major risks (such as paraplegia and death) of the endovascular procedure are akin to those associated with open surgery. 
Thus, patients undergoing such repairs must be cared for in an environment capable of dealing with all such complications. The absence of long-term data for open and endovascular treatment of thoracoabdominal aneurysms must be considered when offering patients any form of intervention. If branched endovascular aneurysm repair parallels its infrarenal or thoracic counterparts, it is likely that the risk of late failure after endovascular techniques is greater than that of open surgery. However, open repair of thoracoabdominal aneurysms is also associated with failure that seems to occur much earlier than open repair of simpler aneurysms. ${ }^{13}$ Therefore, it is incumbent on physicians treating such aneurysms to monitor both groups closely and construct repairs that are amenable to simpler secondary interventions should late failure occur. Recognizing that the techniques described in this article are less than 10 years old, it is not surprising that this procedure is not yet widespread and that longterm data are absent. During this time period, a great deal of device evolution and technique refinement has occurred. In addition, the advances in imaging technology that have paralleled the device improvements will further aid in allowing others to obtain successful results. As clinicians, we can look forward to the results of prospective trials that will likely serve to open our eyes to the risks of both open and endovascular repair in patients with this complex disease.

\section{References}

1. Greenberg RK, Lu Q, Roselli EE, Svensson LG, Moon MC, Hernandez AV, et al. Contemporary analysis of descending thoracic and thoracoabdominal aneurysm repair: a comparison of endovascular and open techniques. Circulation. 2008; 118:808-17.

2. Safi HJ, Estrera AL, Miller CC, Huynh TT, Porat EE, Azizzadeh A, et al. Evolution of risk for neurologic deficit after descending and thoracoabdominal aortic repair. Ann Thorac Surg. 2005;80:2173-9.

3. Coselli JS, LeMaire SA, Conklin LD, Adams GJ. Left heart bypass during descending thoracic aortic aneurysm repair does not reduce the incidence of paraplegia. Ann Thorac Surg. 2004;77:1298-303.

4. Conrad MF, Crawford RS, Davison JK, Cambria RP. Thoracoabdominal aneurysm repair: a 20-year perspective. Ann Thorac Surg. 2007;83:S856-61.

5. Rigberg DA, McGory ML, Zingmond DS, Maggard MA, Agustin M, Lawrence PF, et al. Thirty-day mortality statistics underestimate the risk of repair of thoracoabdominal aortic aneurysms: a statewide experience. J Vasc Surg. 2006;43:217-22.

6. Conway B, Greenberg R, Mastracci TM, Hernandez AV, Coscas R. Renal artery implantation angles in thoracoabdominal aneurysms and their implicatications in the era of branched endografts. J Endovasc Ther. In press.

7. Mohabbat W, Greenberg RK, Mastracci TM, Cury M, Morales JP, Hernandez AV. Revised duplex criteria and outcomes for renal stents and stent grafts following endovascular repair of juxtarenal and thoracoabdominal aneurysms. J Vasc Surg. 2009;49:827-37.

8. Makaroun M, Dillavou ED, Wheatley GH, Cambria RA. Five-year results of endovascular treatment with the Gore TAG device compared with open repair of thoracic aortic aneurysms. J Vasc Surg. 2008;47:912-8.

9. Matsumura J, Cambria RA, Dake M, Moore R, Svensson L, Snyder SA. International controlled clinical trial of thoracic endovascular aneurysm repair with Zenith TX2 endovascular graft: 1-year results. J Vasc Surg. 2008;47:247-57.

10. Morales JP, Greenberg RK, Morales CA, Cury M, Hernandez AV, Lyden SP, et al. Thoracic aortic lesions treated with the Zenith thoracic devices TX1 and TX2: durability and long-term outcomes. J Vasc Surg. 2008;48:54-63.

11. Roselli EE, Greenberg RK, Pfaff K, Francis C, Svensson LG, Lytle BW. Endovascular treatment of thoracoabdominal aortic aneurysms. J Thorac Cardiovasc Surg. 2007; 133:1474-82.

12. Reilly LM, Chuter TA. Endovascular repair of thoracoabdominal aneurysms: design options, device construct, patient selection and complications. J Cardiovasc Surg (Torino). 2009;50:447-60.

13. Coscas R, Greenberg RK, Mastracci TM, Eagleton MJ, Woong CK, Morales C, et al. Associated factors, timing and technical aspects of late failure following open surgical aneurysm repairs. J Vasc Surg. 2010;52:272-81. 